\title{
ZUR LANDSCHAFTS-DISKUSSION IN DER SOWJETGEOGRAPHIE
}

\author{
Constantin v. Regel und Ernst Winkler
}

In den letzten Jahren sind in der sowjetischen Geographie methodologische Diskussionen häufiger und lebhafter geworden. Das hängt offenbar, wie verschiedene sowjetische Erdkundler betonen, daunit zusammen, daß die geographische Wissenschaft hinter der raschen Entwicklung und den Bedürfnissen des Landes zurückblieb, was,von den gleichen Autoren mit der „ungenügenden Klarheit der theoretischen Grundlage der Geographie “ begründet wurde. Die Situation dieser Disziplin in der UdSSR erscheint also trotz anderweitiger Beteuerung über ihre Erfolge als prekär, womit sie ja kaum einen Sonderfall darstellt. Umso aufschlußreicher dürfte es sein, diese Situation einmal kurz zu beleuchten, zumal, wie dieBildung von eigentlichen erdkundlichen Hochschul fakultäten belegt ${ }^{1}$, der Geographie in der Sowjetunion doch einige Wichtigkeit zugemessen wird. Eine solche Orientierung scheint derzeit umso eher möglich, als eine Reihe sowjetischer Arbeiten zum Problem durch vorzügliche Übersetzungen ins Deutsche relativ leicht zugänglich geworden sind ${ }^{2}$.

Eine der aufschlußreichsten Quellen für eine Beurteilung der Lage ist der Bericht der Abteilung für physische Geographie der Geographischen Gesellschaft der UdSSR und des philosophischen Seminars der Geographischen Fakultät der Universität Leningrad, der die Ergebnisse von Sitzungen vom 26. Januar und vom 12. Februar 1951 zusammenfaßt, an welchen sich eine Reihe bekannter sowjetischer Geographen beteiligt hatte ${ }^{3}$. Schon die Eröffnungsworte des durch eine ori-

1 Obgleich der Begriff Fakultät offenbar nicht mit dem westeuropäischen gleichzusetzen ist, weist der Aufbau der sowjetischen Hochschulfakultäten daraufhin, daß die Russen die Geographie bewußt als Fakultät, nicht, wie wir, als (Glied) Disziplin einer Fakultät werten, was nur zur Nachahmung empfohlen werden sollte. (Vgl. E. THIEL: „Die Geographie der Sowjetunion“. Erdkunde II, 1948, Heft 1.) Denn wie jeder Geograph weiß, läßt sich unsere Wissenschaft weder den Natur- noch den Geisteswissenschaften befriedigend einordnen, sondern ist (vielmehr) als eine (wenn auch noch keineswegs ideal verwirklichte) Integration beider, genauer: wesentlicher ihrer Ergebnisse, aufzufassen, ist also in der Tat selbst Fakultät - daneben ja übrigens zugleich sprechender Beweis dafür, daß die Gliederung der Gesamtivissenschaft in Natur- und Geistes (oder Kultur-)wissenschaften mindestens unzureichend ist. Auch deshalb läge es nahe, der Organisation der sowjetischen Geographie besondere Aufmerksamkeit zuzuwenden, und es ist bedauerlich, daß gerade wir Geographen uns die Erfahrungen der Russen nur unzulänglich zunutze machen können (W).

2 Vor allem durch die Zeitschrift "Sowjetwissenschaft “, Berlin $1948 \mathrm{ff}$. (Verlag Kultur und Fortschritt). Vgl. Geographica Helvetica V, 1950, S. 115 und VI, 1951, S. 282.

8 Uber einige theoretische Fragen der physischen Geographie. Nachrichten der Geogr. Gesellschaft der UdSSR 83, 1951, Nr. 3. Dieser Bericht erschien, nachdem unser Aufsatz bereits vorlag (Ende 1951) auch deutsch in "Sowjetwissenschaft “, Naturwissenschaftl. Abt. 5, 1952, 472-498, so daß er nun bequem zugänglich ist. - Um einen Einblick in das weitere methodologische Schrifttum der jüngsten Sowjetgeographie zu geben, seien im folgenden einige Arbeiten zitiert, deren Studium eine vertiefte Orientierung über den Stand der Diskussion ermöglicht. Vollständigkeit ist weder bezweckt noch möglich. Wo nichts anderes vermerkt wurde, handelt es sich um Titel in russischer Sprache. Alampijew, P. M. und Gurari, J. L.: Neue Strömungen in der französischen G. Nachr. d. A kademie d. Wiss. UdSSR G. Ser. 1951. Nr. 2. - Armand, D. L.: Grundlagen der Bilanzmethode in der phys. G. Daselbst 1947, Nr.6; ders.: Funktionale und korrelative Beziehungen in der phys. G. Daselbst 1949. Nr. 1; ders.: Über einige theoretische Grundsätze der phys. G. in Verbindung mit einer kritischen Analyse der Anschauungen des Akademikers A. A. GRIGoRJEw. Daselbst 1951, Nr. 1; ders.: Prinzipien der physisch-g. Rayonierung. Daselbst 1952, Nr. 1; ders.: Die sowjetische G. im Umbruch. Nachr. d. Gesellschaft UdSSR 1952. - BERG, L. S. und Tugarinow, D.: G. Enzyklopädie der UdSSR (deutsch Berlin 1950, II, 1415-1426). - Bonifatjewa, L. I.: Apologie des Imperialismus in der bürgerlichen G am Beispiel einiger Arbeiten über die Ökologie Indiens. Nachr. Akad.d.W.d. UdSSR 1951. - BRozkI!, J. und Michailow, N.: Grundzüge der allgemeinen G von S. W. KALESNIK. G in der Schule, 1948, Nr. 5 (deutsch Sowjetwissenschaft 1949; Nr. 1). - Budyko, M. I.: Zur Intensitätstheorie des phys.-Prozesses. Fragen der G 1949, Nr. 15. Chromow, S. P.: Zur Frage der Methodik der phys. G. Nachrichten der G. Ges. UdSSR 1952, Nr. 1. - Dolgopolow, K.W.: Diskussion des Buches „Der historische Materialismus und die Rolle des g. Milieus bei der Entwicklung der Gesellschaf" "von I. I. Iwanow-Omski (auf der Sitzung des Wiss. Rates d. G. Instituts an der Akad.d. Wiss. d. UdSSR). Nachrichten d. Akad. d. Wiss. UdSSR. G Serie 1951, Nr. 2. - Dumitraschko, N. W. u. a.: Über den gegenwärtigen Stand und die Aufgaben der Geomorphologie. Daselbst 1951, Nr.3. - Grigorjew, A. A.: Einige Ergebnisse der Ausarbeitung 
ginelle "Allgemeine Geographie » bekannten Geographen S. W. KALESNIK ${ }^{4}$ sind so aufschlußreich, daß sie verdienen, im Auszug zitiert zu werden. KALESNIK sagte: «Das Jahr 1950 stellt in der Geschichte der sowjetischen Geographie einen Wendepunkt dar. Die Verordnungen der Regierung über die Errichtung der Wasserkraftwerke an Dnepr und Wolga, der größten der Welt, über den Bau des WolgaDon-Kanals, des Turkmenischen Hauptkanals, des Südukrainischen Kanals und des Nordkrim-Kanals sowie über die Wasserversorgung und Bewässerung des Landes am untern Dnepr, der Krimsteppen, der Bezirke am untern Don, des KaukasusVorlandes, des Landes an der untern Wolga und der turkmenischen Wüsten zeigen der Geographie den Weg, wie sie sich aus einer Wissenschaft, welche die geographischen Landschaften erforscht, in eine Wissenschaft verwandeln kann, die neue geographische Landschaften schafft.... Der Plan zur Umgestaltung der Na-

never Ideen in der phys. G. Daselbst 1946, Nr. 2; ders.: Die großen Stalinschen Bauten und die Aufgaben der G. Daselbst 1951, Nr. 12 (deutsch: Sowjetwissenschaft 1951, Nr. 1). - Isatschenko, A. G.: Die g. Landschaft und ihre kartographische Darstellung. Nachr. G. Ges. UdSSR 1951, Nr. 2; ders.: Die g. Leitgedanken G. N. WYsozkis und sein Beitrag zur g. Theorie. Daselbst 1952, Nr. 1. - Jefremow, G. K.: Die Errichtung eines Erdkundemuseums im neuen Gebäude der Staatlichen Lomonossow-Universität Moskau. Nachr. d. Akad. d. Wiss. UdSSR 1951. Nr. 3. - KalesNik, S. W. Die Bedeutung der Werke Stalins für die G. Nachr. d. G Ges. UdSSR 1950, Nr. 1; ders.: Die Lehre von den Landschaften im Zusammenhang mit der Umgestaltung der Natur in der UdSSR. Nachr. G Ges. UdSSR 1952, Nr. 1; ders.: Sind die Grenzen der Landschaft linear ? Nachr. d. Akad. d. Wiss. UdSSR 1952, Nr. 1; ders.: Was ist unter „Allgemeiner Erdkunde“ zu verstehen ? Nachr. d. G Ges. d. UdSSR 1952, Nr. 1. - Konstantinow, O. A.: Zu den Urteilen A. A. Grigorjews über methodologische Fragen der ökonom. G. Nachr. d. G Ges. d. UdSSR 1952, Nr. 1. - Kotelnikow, W. L.: Die g. Fakultät am Staatlichen W.-I. Lenin-Institut für Pädagogik. Daselbst 1952. Nr. 1. - KuzNECOW, P.S.: Einige Worte über die Bedeutung der vergleichenden Methode in der phys. G. Nachr. d. G. Ges. UdSSR 1950, Nr. 1. - Lidow, W. P.: Aus den Erfahrungen komplexer g. Forschungen (zum Artikel A. N. Smirnows „Über die Grundlagen der G.“) Nachr. d. Akad. d. Wiss. UdSSR 1951, Nr. 2. - LuKaschew, K. I.: Imperialistische Konzeptionen in der heutigen amerikanischen Pseudog. Daselbst 1952, Nr. 1 (deutsch Zeitschrift für Geopolitik 1952, Nr. 10). - MAKEJEw, P. S.: Über G, natürliche Zonen und Landschaften. Nachr. d. G. Ges. UdSSR 1951, Nr. 12 (deutsch in „Sowjetwissenschaft" Naturwiss. Abt. 1952, Nr. 2). - Markow, K. K.: Über die Bedeutung der historischen Methode in der G. Daselbst 1947, Nr. 1; ders.: Irrtümer des Akademiemitgliedes A. A. GrIGoRJEw. Daselbst 1950. Nr. 12 (deutsch in "Sowjetwissenschaft“ 1951, Naturwiss. Abt. Nr. 4). - Milkow, W. L.: Zur Analyse der Landschaftsgrenzen auf der russischen Ebene. Nachr. d. Akad. d. Wiss. UdSSR 1952, Nr. 1. - Nalivikin, D. W.: I. W. Muschketow und die g. Geologie. Nachr. d. G. Ges. UdSSR 1952, Nr. 1. - Nikitin, N. P.: Die Entstehung der Wirtschaftsg. in Rußland. Fragen der G. 1950, Nr. 17. - Organisationsprinzipien und Methodik komplexer g Forschungen. Nachr. d. G Ges. UdSSR 1952, Nr. 1. - PANow, D. G.: Über die Unterwasser-Landschaften des Weltmeeres. Nachr. G Ges. UdSSR 1950. - Pomus, M. I.: Die Behandlung der Fragen der Bevölkerungsg in der Literatur der ökonom. G. Moskau 1950. - SABelin, I. M.: Einige Bemerkungen zum Artikel von A. M. Smirnoiv. Nachr. Akad. d. Wiss. UdSSR 1952, Nr. 1. - Sauschkin, J. G.: Die Grundidee der russischen klassischen G vor der Oktoberrevolution. Fragen der G. 1948, Nr. 9; ders.: Über einige Fragen der Wechselwirkung zwischen Gesellschaft und $\mathrm{g}$ Milieu im G-Unterricht der Schulen der UdSSR. Die G in der Schule 1949, Nr. 2; ders.: Über den Artikal „Zur Frage nach dem Gegenstand der ökonomischen G als Wissenschaft “ von M. M. SchiRmUNski. Nachr. d. Akad. d. Wiss. UdSSR 1952, Nr. 1. - SchiRmUnSKi, M. M.: Die Militarisierung und ideelle Zusammensetzung der bürgerlichen amerikanischen G. Daselbst 1952, Nr. 2. - Schiwaco, A. W.: Zum Artikel D. G. Panows „Über die unterseeischen Landschaften des Weltmeeres“. Daselbst 1951, Nr. 2. - SMIRNow, A. M.: Über fehlerhafte Auffassungen in theoretischen Fragen der G. Nachr. d. Ges. UdSSR 1951, Nr. 3 (deutsch in "Sowjetwissenschaft 1951, Naturw. Abt. Nr. 4. - SolnzEw, N. A.: Die natürliche $\mathrm{g}$ Landschaft und gewisse allgemeine Gesetzmäßigkeiten derselben. Arbeiten des 2. Unionskongresses d. G. I, 1948, 212 ff.; ders.: Über die Morphologie der natürlichen g Landschaft. Fragen d. G. 1949, Nr. 16. - Stalins Arbeit "Marxismus und Fragen der Sprachwissenschaft" und die Aufgaben der ökonomischen G. Nachr. d." G Ges. UdSSR 1951, Nr. 2. - StschukIN, I. S.: Gedanken über Wesen und Methodik der komplexen physisch-g. Rayonierung. Fragen der G. 1947, Nr. 3. - WassiLjEw, K.: Losgelöst vom Leben (Kritik der Theorie des g Prozesses bei A. A. Grigor!ew) Kultur und Leben 1950, Nr. 9. - W VEDENSKI, N. W.: Über einige Fragen der phys. G. Nachr. G Ges. 1952, Nr. 1. - Der 19. Parteitag der KPdSU und die Aufgaben der Sowjetg. Nachr. G Ges. UdSSR 1952, Nr. 6. Die Aufgaben der G Gesellschaft im Zusammenhang mit den Beschlüssen des 19. Parteitages der KPdSU. Nachr. G Ges. UdSSR 1952, Folge 6.

4 Grundzüge der allgemeinen Geographie. Moskau-Leningrad 1947, 483 S. Vgl. die Besprechung von J. BRozKIJ und N. Michallow in Sowjetwissenschaft 2, 1949, 229-243. 
tur, der jetzt in unserm Lande realisiert wird, ist sowohl ein Akt höchsten Vertrauens $\mathrm{zu}$ den Geographen der Sowjetunion als auch eine ernsthafte nachdrückliche Mahnung daran, daß es für die Geographie an der Zeit ist, sich so umzustellen, daß sie nicht hinter dem Tempo unseres Lebens und hinter den Ansprüchen der Volkswirtschaft zurückbleibt. Die entschiedene Umstellung des Interesses der Geographen auf den Dienst an den volkswirtschaftlichen Maßnahmen erfordert zugleich, die geographische Theorie so klar wie möglich zu gestalten. Von der gewaltigen Bedeutung einer fortschrittlichen wissenschaftlichen Theorie zeugen die neuen genialen Arbeiten des Genossen Stalin, die der Sprachwissenschaft gewidmet sind. Vor den Arbeiten des Genossen Stalin herrschte in der Sprachwissenschaft.. Verwirrung. Genosse Stalin hat durch seine Arbeiten das Fundament für die Sprachwissenschaft gelegt und zugleich die Ursachen jener Verwirrung und Stagnation aufgedeckt, die in der Sprachwissenschaft herrschten... Es muß ohne Vorbehalt gesagt werden: Das, was wir jetzt in der geographischen Theorie haben, ist bekanntermaßen ein Spiegelbild dessen, was in der Sprachwissenschaft zu der Zeit war, die dem Erscheinen der genialen Arbeiten des Genossen STALiN vorausging ${ }^{5}$. Was sind Gegenstand, Umfang und Inhalt der physischen Geographie? Was ist eine geographische Landschaft und welches sind ihre Besonderheiten? Was ist der geographische Prozeß? . . . Selbstverständlich hat jeder Geograph Antworten darauf bereit: Es wäre seltsam, wenn die Leute, die sich mit der Geographie beschäftigen, sich über diese Probleme keine bestimmte Ansicht gebildet hätten. Aber das Schlimme ist, daß die Antworten bei verschiedenen Personen verschieden ausfallen - ein sicheres Zeichen dafür, daß ein Teil der Antworten fehlerhaft ist. Die Ursache ist dieselbe wie bei den Sprachwissenschaften. Es gibt keine oder fast keine ausführlichen und freien Gespräche, weder auf den wissenschaftlichen Sitzungen der geographischen Institutionen noch in der geographischen Presse.... Erst in den letzten zwei Monaten haben sich im Zusammenhang mit dem Studium der genialen Arbeiten des Genossen STALiN über die Sprachwissenschaft in der Geographischen Gesellschaft Diskussionen zur Theorie einer Anzahl geographisch-wissenschaftlicher Fragen entfaltet. Die Arbeiten des Genossen Stalin . . zeigen, daß man jede beliebige komplizierte Idee klar und einfach ausdrücken kann. Zahlreiche Geographen folgen diesem Vorbild nicht. Es ist keine Seltenheit, in geographischen Aufsätzen eine komplizierte Terminologie (mit Begriffen wie «psychral», hyperpsychral » usw.) anzutreffen, und es herrscht eine Flut von Fremdwörtern... Ferner liegt oft eine durch nichts gerechtfertigte Tendenz vor, bereits in der Geographie existierenden Begriffen immer neue Bezeichnungen zu geben; als Beispiel kann der Begriff der Landschaft dienen, den die verschiedenen Autoren mit den Bezeichnungen «Landschaft», «Geochore », «Aspekt», «Geozönose » usw. belegen. Es liegt indes keinerlei Bedürfnis dafür vor, das Ergebnis aber ist eine ziemliche Verwirrung ${ }^{6}$, weil das Auftreten eines neuen Fachausdruckes gewöhnlich gleichbedeutend mit dem Auftreten eines neuen Begriffes ist, während tatsächlich in einer Reihe von Fällen der neue Fachausdruck nur einen alten Begriff auf andere Weise bezeichnet. Eine der schlimmsten kranken Stellen in unserer Arbeit ist jedoch, wic gesagt, die mangelhafte Entwicklung theoretischer Diskussionen. Infolgedessen mangelt es an gegenseitigem Verstehen selbst inbezug auf gewisse Grundthesen der geographischen Theorie ».

Kalesnik geht dann auf Angriffe ein, die A. M. Smirnow sowohl gegen die ausländische bürgerliche (vor allem gegen A. HETTNER) als auch gegen die sowjetische Geographie gerichtet hatte und in der diese letztere (nach S.MIRNow in drei

6 Wenn auch seither die personelle Situation geändert hat, dürfte davon die durch ,den dialektischen Materialismus charakterisierte ideologische kaum berührt worden sein.

' Die beinahe gleiche Formulierung findet sich bezeichnenderweise bei R. HaR'TSHORNE, "The Nature of Geography“, Lancaster PA, 1939 (und 1951), der sogar ein Kapitel seines Buches mit „The present confusion “ überschrieb. 
Schulen: diejenige L. S. Bergs, die Schule von Moskau und diejenige des Akademikers A. A. GRIGORJEws zerfallend) im wesentlichen als idealistisch und metaphysisch taxiert worden war - wohl die schwerste Insinuation, die gegen einen Marxisten zu machen ist. SMIRNows Vorwürfe galten vor allem der «vollkommen falschen 》 These von der Landschaft, die nach ihm ein «pseudowissenschaftlicher Begriff..., keine objektiv existierende Realität», sondern etwas 《Erdachtes» ist.

SMIRNow begründete seine Ansicht zur Hauptsache mit folgenden (unbewiesenen, aber freilich ebenso schwer beweis- wie widerlegbaren) Behauptungen: 1. die Landschaft habe «nichts mit... wirklichen Komplexen der Natur gemein »..., sei «eben nur «Paysage», d. h. äußeres Bild der Natur in willkürlichen Abgrenzungen (kursiv durch uns); 2. das Zusammentreffen der Objekte und Erscheinungen in territorial begrenzten Gebieten sei faktisch zufällig ${ }^{7}$, d. h. die Landschaft sei lediglich ein Agglomerat von realen Erscheinungen (da nämlich einzig und allein die Natur als Ganzes einheitlich und unteilbar sei); 3. die Landschaft werde ferner losgelöst von der Gesamtheit des geographischen Milieus (der gesamten Erdhülle) betrachtet, wodurch dieses letztere zur einfachen Summe willkürlicher, von Ort zu Ort verschiedener Verbindungen sich gegenseitig beeinflussender Faktoren und ihrer Äußerungen gestempelt werde, schließlich 4. die Landschaften würden von den «metaphysischen Landschaftskundlern» nach dem Prinzip der Gleichartigkeit der Faktoren gesondert und klassiert (typisiert), während das geographische Milieu tatsächlich mannigfaltig sei (aus formal wie material verschiedenen Erscheinungskomplexen bestehe), und außerdem befänden sich die so gefundenen Klassifikationseinheiten in schreiendem Widerspruch zu den Interessen der Volkswirtschaft, weil ein wirtschaftlich organisiertes Territorium nicht gleichartige, sondern in natürlicher Hinsicht verschiedenartige (räumlich-material-formale) Teile umschließe. Diese kritischen Thesen Smirnows suchte Kalesnik zu entkräften, indem er betonte: "Die Behauptung Smirnows, die Landschaft sei etwas Erdachtes, entspricht nicht der Wahrheit. Die Erdoberfläche (im Sinne des Geographen) ist... ungewöhnlich mannigfaltig. Doch kann sich eine bestimmte Kombination von Elementen... von Ort zu Ort wiederhölen und schafft so eine natürliche Landschaft, die eine objektive Realität und nicht das Produkt der Verallgemeinerung des menschlichen Verstandes ist ». Den Beweis für diese Gegenthese sucht KALESNiK an konkreten Beispielen zu erbringen: "Nehmen wir an, daß der Forscher in einer Gegend folgendes sieht: Hügel, zwischen . . Senken, die von Sümpfen oder Seen erfüllt sind; Nadelwald auf den Hügeln und Mischwald in den Senken, soweit diese nicht die Seen einnehmen; Podsolböden. Auf seinem weitern Wege überzeugt er sich, daß diese charakteristische Kombination ... . sich wiederholt.... Er überzeugt sich ferner davon, daß der Augenblick kommt, in dem die beschriebene Kombination von einer andern abgelöst wird, und diese andere sich ebenfalls regelmäßig über Dutzende von Kilometern hin wiederholt und ein neues, in seinen Zügen anderes 'T'eilstück bildet. Diese sich voneinander unterscheidenden Teilstücke, Landschaften (d. h. Kombinationen bestimmter Litho-Atmo-, Hydro- und Biosphärenstücke, W.), kann jeder sehen, begreifen und erforschen, denn sie existieren wirklich in der Natur» (kursiv von uns). Die weitere Behauptung Smirnows, daß die «Landschaft ein zufälliges Zusammentreffen von Phäno-

7 wobei freilich nicht klar gemacht wird, welche der verschiedenen Bedeutungen von "zufällig" gemeint ist. "Zufällig“ ist die Landschaft zweifellos in dem Sinn, daß ihre "Primärursache“ nie zu eruieren sein wird. „Zufällig“ ist sie aber auch, insofern (ihre) „Notwendigkeit" nicht absolut beweisbar ist, endlich insofern sie (wie ihre Teile) veränderlich (d. h. keine absolute, unbeschränkte Dauer hat) und nicht (absolut) berechenbar ist. Aus diesen Bedeutungen indes „Irrealität", „Wesenlosigkeit", "Sinnleere“ der Landschaft abzuleiten, würde konsequenterweise bedingen, mit sämtlichen realen Objekten dasselbe zu tun, da für alle die gleichen Existenzmomente gelten. Und jedenfalls würde davon auch die Erforschbarkeit nicht berührt, da Wissenschaft selbst vor dem „Irrealen“ nicht Halt machen "darf " $"(W$. 
menen 》 sei, suchte KALESNIK dadurch zu widerlegen, daß er betonte, die Erfahrung lehre die Natur als «zusammenhängendes, einheitliches Ganzes 》, die Objekte und Erscheinungen in organischem Zusammenhang und wechselseitiger Bedingung befindlich erkennen, und sie treibe die Wissenschaft, nach ENGELs, « diesen systematischen Zusammenhang überall im einzelnen wie im ganzen nachzuweisen ». Wohl sei klar, daß «man eine echte Gesetzmäßigkeit der Erscheinungen nicht feststellen (könne), wenn man sie nur in territorialer Kombination (betrachte), da die Landschaft kein autonomes System, sondern nur Teil eines größern Ganzen: des gesamten geographischen Milieus 》 sei ${ }^{8}$. "Aber die örtlichen Zusammenhänge innerhalb der Landschaft rein äußerlich zu nennen und zu behaupten, daß sie zufällig seien, das (heiße) einen schweren methodologischen Fehler begehen und eine metaphysische Position beziehen... Ist es wirklich zufällig, daß die Kiefer nicht auf Schwarzerde wächst, daß der Fluß im Tale fließt und nicht auf dem Kamm der Wasserscheide, daß es auf den Südhängen der Hügel wärmer ist, als auf den Nordhängen? Sind denn Bau und Funktionen des Herzens allein darum zufällig, weil es ein Teil des Organismus ist und eine «territorial begrenzte 》 und dabei einheitliche Kombination von Zellen und Geweben darstellt?... Fälschlich und unbegründet 》 sagt KALESNIK weiter, beschuldige SMIRNOw «alle sowjetischen Geographen, daß sie die Landschaft losgelöst vom geographischen Milieu als Ganzem betrachtet ». KaLESNIK zitiert in diesem Zusammenhang einen eigenen Satz

8 Auch zu behaupten, Landschaft sei kein „autonomes System “, ist ein mindestens fragwürdiges Unterfangen. Ist denn etwa der Mensch, der Kosmos, autonom, d.h. sich völlig selbst bestimmend? Das Autonomieproblem hängt unmittelbar zusammen mit dem von den Sowvets der „bürgerlichen“ Geographie nicht selten gemachten Vorwurf, sie pflege (verwerflichen) Determinismus. Er wird zumeist an den Stalinschen Satz geknüpft, daß das geographische Milieu zwar „eine der ständigen und notwendigen Bedingungen der Entwicklung der Gesellschaf“ sei und „deren Entwicklungsgang beschleunige oder verlangsame“, daß aber „sein Einfluß nicht bestimmend “ sei, wobei bestimmend wohl besser mit (allein) entscheidend übersetzt würde (J. Stalin: Fragen des Leninismus, Moskau 1947, p. 548). Da der Vorwurf deterministischen Denkens ja dann und wann auch bei uns im „Westen“ zu hören ist, wobei vor allem F. RATZEL bezichtigt wird, ihm Vorschub geleistet, wenn nicht sogar es begründet zu haben (C.'TRoll: Die geogr. Wissenschaft in Deutschland ... Erdkunde I, 1947, p. 23; H. BOBEK: Stellung und Bedeutung der Sozialgeographie. Erdkunde II, 1948, p.119; H. ONDE: Géographie et civilisation. Le Globe LIII, VII, 1948, p. 89, der jedoch offenbar nur dem längst widerlegten L. FÈBVRE: La Terre et l'évolution humaine, Paris 1922 nachschrieb, sowie zahlreiche andere), darf hier gewiß wieder einmal daraufhin gewiesen werden 1. daß die Autonomie des Menschen doch wohl ausschließlich eine „Rahmen"freiheit, ein Selbständigsein in gewissen durch Erde (Gravitation, Biologie: Atmung, Nahrungsgebundenheit, Geburt, Tod usw.), Kosmos (Solarstrahlung u.a.) und ev. „übernatürliche " Kräfte gebotenen Grenzen darstellt, 2. daß diese Rahmenfreiheit schon ein RATZEL nachdrücklich vertreten hatte (z. B. in Anthropogeographie 1882, p. 51: „.. es gibt (im Bereich des Menschlichen) keinen Zwang, kein unbeugsames Gesetz, sondern es sind weite Grenzen, innerhalb deren der Mensch seinen Willen, ja selbst seine Willkür zur Geltung zu bringen vermag. Und dies ist es eben, was alle Studien über den Zusammenhang zwischen Geschichte und Naturumgebung so erschwert, daß wir allgemeine Schlüsse nur immer bedingungsweise aussprechen können. Der eine Faktor in diesem Zusammenhang... ist eben nicht berechenbar..., weil er frei ist; es ist dieses der menschliche Wille"), 3. aber da $B$ diese These von der relativen Freiheit des Menschen auch in HetrNers ins Russische übersetztem Hauptwerk „Die Geographie, ihre Geschichte, ihr Wesen und ihre Methoden“ (Breslau 1927, p. 209, 267 u. a. a. O., wo deutlich gesagt ist, daß a) Determinismns und Indeterminismus sich gar nicht a priori fixieren lassen, b) da $\beta$ Determinismus immerhin eine bisher unwiderlegte Hypothese der Wissenschaft sei, er aber c) keineswegs bedeute, daß jeder Mensch auf das gleiche Motiv gleich antworten müsse, die Entscheidung... vielmehr immer vom Charakter und der ganzen Vorgeschichte des Menschen" abhänge, wobei auch der Nachsatz nicht unterdrückt werden soll: [der Mensch] „wird durch die Annahme der Bestimmtheit seines Willens nicht von der sittlichen Verantwortung entbunden, er haftet mit seiner ganzen Persönlichkeit für sie“) unmißverständlichen Ausdruck erhalten hatte, so daß die Interpretation dieses Determinismus durch die Sowjets mindestens merkwürdig anmuten muß. Vgl. in diesem Zusammenhang die kritische Stellungnahme von V.S. Radovanović „Au sujet d'une nouvelle contribution à la question des changements et du développement du milieu géographique" (Bulletin de l'Institut Ethnogr. de l'Académie serbe des sciences 1, 1952, Nr. 1-2) zu den Ausführungen von V. L. Kotelnikoff „Über die durch die agrikole Tätigkeit des Menschen bedingten Handlungen des geographischen Milieus." Nachr. G. Ges. UdSSR 82, 1950. (W.) 
aus dem Jahre 1947: «Wie die geographische Landschaft von der geographischen Sphäre (Milieu als Ganzes) untrennbar ist und einen Teil desselben darstellt, ist auch die geographische Sphäre untrennbar von der Landschaft, da sie in den Landschaften und durch sie sichtbar wird... Die geographische Landschaft ist ein Teilstück der geographischen Sphäre, das eine bestimmte Individualstruktur besitzt (also Einheit im Sinne eines Wirkzusammenhanges, W.), die über einen größern Raum typisch ausgeprägt ist, unmittelbar mit der Struktur der geographischen Sphäre als Ganzem zusammenhängt und von ihr bedingt ist ». Die Frage der Landschaftsklassifikation endlich, glaubt KALESNIK mit dem Hinweis darauf lösen zu können, daß es in der Wissenschaft überhaupt üblich sei, Objekte und Erscheinungen nicht nach ihrer Verschiedenheit, sondern nach dem Grade ihrer Ähnlichkeit zu Gruppen zusammenzufassen. «Die Gruppen der Fette, Eiweißstoffe und Kohlenhydrate umfassen Verbindungen, die sich durch eine gewisse Gemeinsamkeit des Baues auszeichnen. . . Den Bodenkundlern ist es nie in den Sinn gekommen, in ihren Klassifikationen die Podsole mit den Schwarzerden, und den Biologen nicht, die Flossenfüßler mit den Blattkiemern zusammenzufassen. Allerdings handelt es sich in den genannten Fällen um Systematik und nicht um territoriale Gliederung. Aber auch bei letzterer ist «Einheitlichkeit», worunter man nur die typische Wiederholung bestimmter Kombinationen ungleichartiger Objekte... zu verstehen hat, die Grundbedingung für die Fernhaltung von Willkür aus der Klassifikation. Die Grenze zwischen den Rayons verläuft da, wo ein Gleichartigkeitstyp höherer oder niederer Ordnung (oder auch nur verschiedener Komplexität, W.), von einem andern Gleichartigkeitstyp verdrängt wird ».

Nach diesen apologetischen Erörterungen geht KALESNIK zum Gegenangriff auf SMIRNOw über, dem er seinerseits entgegenhält, widerspruchsvolle und inkonsequente Gedankengänge gepflegt zu haben. Vor allem wirft er ihm vor, daß seine Ansicht vom ganzheitlichen und gesetzmäßigen geographischen Milieu als dem eigentlichen Objekt der Geographie weder neu sei (sondern, ohne daß SMIRNow dies anzumerken für nötig erachtet habe, die Auffassung der meisten Sowjetgeographen spiegle), noch die Möglichkeit und Notwendigkeit der besondern Erforschung der Landschaftsteile ausschließe. «Die Erdoberfläche ist viel zu groß (und zu komplex, W.), als daß sie bei der Erforschung auf einmal erfaßt werden könnte, und wie die Erforschung des Atoms nicht etwa einen Verzicht auf den Begriff der Ganzheit der Materie bedingt, so bedeutet die Erforschung der Landschaften nicht den Verzicht auf den Begriff der Ganzheit des geographischen Milieus. Die Vorstellungen von den allgemeinen Gesetzmäßigkeiten des geographischen Milieus gehen in ihrer Bildung und . . Vervollkommnung nur den einen Weg: Die regionalen Forschungen werden erweitert und vertieft, die Resultate.. werden verglichen, und das Spezielle wird vom Allgemeineren gesondert, wobei man sich klar ist, daß die untersuchten Landschaften nur Teilstücke des gesamten geographischen Milieus und die Besonderheiten ihres Baues und ihrer Entwicklung im Grunde nur die Konkretisierung der allgemeinen Gesetzmäßigkeit des Baus und der Entwicklung der gesamten Erdoberfläche sind». Einen wesentlichen Widerspruch sieht KALESNIK sodann darin, daß SMIRNow als wichtigste geographische Kategorie die geographische Zone aufstellte, die nach ihm gebietsmäßig nicht untergliedert werden könne, während er selbst doch zwischen- und innerzonale Gruppierungen der terrestrischen Phänomene unterscheide, denen er die Bezeichnung «geographische Blöcke» verliehen und die er sogar weiter, bis hinab $\mathrm{zu}$ den «wirtschaftlich organisierten Gebieten 》 des einzelnen Kolchos und Sowchos (also [Groß-]Betrieb) unterteilt habe. "SMIRNOw beschuldigt die übrigen Sowjetgeographen der Willkür bei der Rayonierung, hat aber selbst.. ein noch willkürlicheres und noch weniger begründetes Schema vorschlagen können ». Schließlich kritisiert KaLESNIK scharf die Meinung von Smirnow, das Spezifische der Geographie bestehe darin, «daß das geo- 
graphische Milieu als Vorbedingung für das Leben der Gesellschaft studiert werden » müsse, d. h. «nicht aus der Position der < reinen> Wissenschaft, sondern als Objekt organisierter und planmäßiger Einwirkung seitens unserer sozialistischen Gesellschaft ».. 《Zwar sei, sagt KaLESNIK, der Gedanke an sich unbestritten, insofern jede Wissenschaft der Sache des Kommunismus dienstbar zu machen 》 sei. Darin liege daher gar kein «Spezifikum» der Geographie. SMIRNow habe indes die Aufgabe der Geographie mit ihrem Ziel verwechselt. «Die Aufgabe der Geographie ist die Erforschung des gesamten ganzheitlichen und .... gesetzmäßig sich entwickelnden geographischen Milieus. Wohl aber ist das Ziel der Erforschung die Ausnutzung dieses Milieus im Interesse einer beschleunigten Entwicklung der sozialistischen Gesellschaft ».

Zum Abschluß seiner Einführung hielt KalesNik fest, daß Smirnows Erörterungen «nur einen Einzelfall in der Reihe der theoretischen Äußerungen 》 darstellten, und daß es «längst Zeit 》 (sei), von der Gesamtheit der Fehler und positiven Leistungen der sowjetischen geographischen Theorie (zu) sprechen, (wobei) natürlich in erster Linie von den Fehlern zu reden sei. «Erinnern wir uns daran, daß die Lehre von den Landschaften eine Reihe wesentlicher Mängel besitzt. Wenn auch Hettners Klassifikation der Wissenschaften (in Sach-, Zeit- und Raumwissenschaften und die Zuordnung der Geographie zu letzteren, W.) von den sowjetischen Geographen längst verurteilt worden ist und als solche nur in ihrer harten und scharfen Kritik Erwähnung findet, macht sich in zahlreichen geographischen Werken das Wesen dieser Klassifizierung doch immer noch weiter bemerkbar ${ }^{9}$. Viele geographische Darstellungen bestehen noch aus isolierten Angaben über Relief, Klima, Böden usw. ohne den Versuch, diese Angaben zu einem Ganzen zu vereinigen. Die Elemente der Landschaft liegen gleich Schubfächern nebeneinander, und dadurch erweisen sich Landschaft und Land nicht als etwas innerlich Ganzes und Einheitliches, sondern nur als ein Raum, der mit Relief, Klima usw. angefüllt ist (kursiv von uns). Sieht man die Angelegenheit von dieser Seite an, so erhält man doch auch objektiv ein Abbild des Hetronerschen chorologischen Prinzips. Die meisten.. Werke beschreiben (ferner) bisher nicht wirklich existierende, sondern « rekonstruierte» Landschaften, d. h. solche, die von dem Stempel, den ihnen die Tätigkeit des Menschen aufgedrückt hat, «befreit» sind. Daher « rauschen die Federgrassteppen 》 noch bis heute im Süden der Ukraine, die es dort längst nicht mehr gibt. Die Beschreibung der Natur (landschaft, W.) ohne die gehörige Berücksichtigung der Einwirkung des Menschen... ist ein Fehler, der speziell auch meiner Arbeit «Nordkaukasien und der untere Don 》 eigen ist. (Weiterhin) erkennen gewisse Landschaftskundler die allgemeine Erdkunde nicht als geographische Disziplin und selbständige Wissenschaft an. Und doch bedeutet auch das, was SMIR Now sagt: Loslösung der Landschaft vom gesamten geographischen Milieu (KALESNIK versteht unter allgemeiner Erdkunde: die Lehre von der Gesamtheit aller Landschaften oder vom geographischen «Gesamtmilieu», zugleich aber auch die Lehre von den Gesetzmäßigkeiten, die diese Erscheinungen beherrschen; or faßt also [gesamthaft] individuelle und generelle Betrachtung zusammen, W.) ${ }^{10}$. Ich

9 Ein anderer Sowjetgeograph, A. A. Grigorjew, äußert sich über die Wirkung HettNers in nicht minder bezeichnender Weise (Die großen Stalinschen Bauten und die Aufgaben der Geographie. Bote der Akademie der Wissenschaften der UdSSR 1951, Nr. 12, deutsch in Sowjetwissenschaft 4, 1951, Naturwiss. Abt., Heft 1): „Es ist hervorzuheben, daß sich die Träger der Ansichten (von der Landschaft) früher als Nachfolger des deutschen Geographen HETTNER bezeichneten; heute werden solche Anschauungen als angeblich neue Ideen in der Sowjetgeographie propagiert “.

10 Dies taten und tun auch, westliche " Geographen, so A. HeTtNer, H. Schmitrhenner u. a. ausdrücklich, indem sie unter allgemeiner Geographie vergleichend-nomothetische Betrachtung der Landschaften und Länder + (individuelle) Betrachtung der Gesamterdoberfläche verstehen. Ob sich diese "Kombination" logisch halten läßt, bleibe hier dahingestellt. Nur darauf ist hinzuweisen, daß sowohl eine idiographische (individuelle) als auch eine nomothetische (generelle) Betrachtung der Landschaft im Sinne eines Ausschnittes der Erdhülle und der terrestrischen Hülle selbst im 
habe mich bekanntlich bemüht, die Wichtigkeit der allgemeinen Erdkunde zu beweisen. Leider stehen längst nicht alle Geographen auf diesem Standpunkt. Darin ist offenkundig auch die Tatsache begründet, daß die allgemeine Erdkunde aus den Lehrplänen der geographischen Fakultäten herausgenommen wurde. (Schließlich) weist auch die 'Theorie vom physisch-geographischen Prozeß wesentliche Fehler auf..... (vor allem zwei grundlegende) 1. die Auffassung des physisch-geographischen Prozesses als einer nur einmaligen Wechselwirkung der Objekte... und 2. die Charakteristik dieses Prozesses als eines Dirigenten, der außerhalb des geographischen Milieus, über demselben steht » (während, auf Grund der Hauptprinzipien des dialektischen Materialismus der Prozeß eine Erscheinungs « form 》 der Materie, also der Landschaft selbst ist, da, nach ENGELs, «Materie ohne Bewegung ebenso undenkbar ist wie Bewegung ohne Materie», W.). Schlecht sei aber vor allen Dingen, so endete KALESNIK, daß in der sowjetischen Geographie überhaupt 》 zwei theoretische Richtungen existieren. Die sowjetische geographische Theorie soll einheitlich sein wie unsere materiliastische, marxistisch-leninistische Weltanschauung... Diese einheitliche Theorie müssen wir auch im Feuer gegenseitiger kameradschaftlicher Kritik schmieden, gestützt auf die geographische Praxis und die grundlegenden Arbeiten der Klassiker des Marxismus, insbesondere auf die genialen Arbeiten des Genossen Stalin 》.

In ähnlicher Richtung wie KALESNIK motivierten zumeist auch die übrigen Teilnehmer des eingangs genannten methodologischen Gespräches ihre Stellungnahmen, so daß jener in seinem Schlußwort feststellen konnte, die Diskussionsbeiträge lägen «mehr oder weniger in einer Linie » ${ }^{11}$. Ein näheres Eingehen auf sie erübrigt sich deshalb. Es seien denn auch nur noch die Voten erwähnt, welche Ergänzungen zu denjenigen KALESNIKS darstellen.

Als eine solche kann die These A. G. IsATSchenkos betrachtet werden, die Landschaft sei, wenn man sich so ausdrücken dürfe, eine «einheitliche Uneinheitlichkeit 》, d. h. - was übrigens schon KALESNIK mit dem Hinweis auf die Forderung, die Landschaft in «echt dialektischem Aspekt» als zugleich uneinheitliches und einheitliches Territorium aufzufassen, angedeutet hatte - ein Gebilde, das sich zwar aus ungleichartigen Bestandteilen: Boden, Lufthülle, Organismen usw. zusammensetze 12, aber infolge der intensiven Wechselwirkung dieser Erscheinungen, ihrer Kombination, und deren Wiederholung im Raume (wie übrigens auch in der Zeit, W.) gleichzeitig einen Wirkzusammenhang = Einheit bilde, der allerdings in unaufhörlicher Wandlung («Selbstentwicklung 》) begriffen sei. Daher sei denn auch die Theorie der Landschaft (in der Sowjetgeographie) auf drei Grundlinien: der Struktur, der räumlichen Verbreitung und der zeitlichen Entwicklung der Landschaft aufzubauen, wie schon KaLESNIK gefordert habe. Hinsichtlich des «geographischen Prozesses》 betonte Isatschenko, der zwei gegnerische Richtungen der Geographie: die «Landschaftsrichtung 》 und die «Prozeßrichtung 》 unrerschied, daß es «kein Studium der geographischen Landschaft ohne Erforschung des geographischen Prozesses geben » könne, ein Gegensatz zwischen einer nur statischen und einer nur dynamischen Forschung widersinnig sei. Schließlich sieht IsATSCHENko die bisher bestehende Unzulänglichkeit der Geographie bei der Lösung praktischer Aufgaben im Fehlen geographischer Spezialisten begründet, und er fordert deshalb die Ausbildung solcher: «In der Botanik sind rund 500000 Pflanzenarten bekannt. Ich erinnere mich der Äußerung eines großen Botanikers, der

Sinne der Gesamtheit der Landschaften (d. h. der „Globallandschaft “) möglich und nötig ist. Im Interesse klarer Handhabung der Begriffe läge es aber zweifellos, wenn in diesem Zusammenhang allgemeine Geographie ausschließlich mit genereller Betrachtung gleichgesetzt würde.

$11 \mathrm{Ob}$ dies der Autorität KALEsNiks zuzuschreiben ist, entzieht sich unserer Kenntnis, hat aber im betrachteten Rahmen nur akzessorische Bedeutung.

12 also in jedem Fall heterogen, nicht homogen ist (wie gewisse westliche Geographen anzunehmen scheinen). 
gesagt hat, daß er etwa 2000 ... kennt; ein durchschnittlicher Botaniker kennt also sicher weniger. In der Biologie sind hervorragende Fachleute bekannt, die ihr ganzes Leben lang auf einem bestimmten <engen> Gebiet arbeiten, das nur ein kleines Stück im System der betreffenden Wissenschaft umfaßt. In der Geographie haben wir keine solche Differenzierung, und das stellt ein großes Hemmnis dar... warum sollte man sich nicht auf dem Gebiet eines Landschaftstyps, eines Formentyps usw. spezialisieren? Das würde die Arbeit des Geographen erleichtern und auf realen Boden stellen. Bei uns empfängt jeder spezialisierte Geograph die gleiche Vorbildung... Man soll ihn in die Tundra schicken; dort wird er auf konkrete Probleme stoßen, z. B. Renweiden, Ackerbau im Polargebiet usw. Wenn ein Mensch in die Wüste fährt, steht er dort vor völlig andern Aufgaben... Weil nun vor unserer sozialistischen Gesellschaft bestimmte Probleme unter (räumlich) verschiedenen.. Bedingungen auftauchen.., muß man das auch bei der Ausbildung spezialisierter Geographen berücksichtigen ».

In die gleiche Kerbe spezialisierter und vor allem am praktischen Beispiel orientierter und für die Praxis, d. h. für die Gesellschaft arbeitender geographischer Forschung schlug N. W. KRYLOW, wenn er verlangte, die gesamte geographische Arbeit noch mehr in den Dienst des Lebens zu stellen. "Die Geographie stellt die allgemeinen Entwicklungsgesetze der geographischen Sphäre der Erde fest, untersucht ihre Umgestaltung in der Geschichte der ... Gesellschaft und arbeitet die natürlichen und gesellschaftlichen Grundlagen für eine wissenschaftliche geographische Prognose aus 》. Hier klingt ein neuer Gesichtspunkt an, der in der Sowjetgeographie der letzten Jahre zu gleichfalls scharfen Auseinandersetzungen geführt hatte: zum Gegensatz einer physischen und einer ökonomischen Geographie. «Die gesamte vorausgegangene Entwicklungsperiode der physischen Geographie der Landschaft und der Wirtschaftsgeographie des Rayons hat uns jetzt an die Feststellung der gemeinsamen Aufgabe für (beide) herangeführt, die bisher faktisch nebeneinander bestanden haben... (Ihre Lösung wird dann erfolgen können) ... « wenn die Wissenschaften sich gemäß der Klassifikation von ENGELS nicht nur nach dem Merkmal der Analyse irgendeiner einzelnen Bewegungsform der Materie gliedern, sondern auch nach dem Prinzip zusammengehöriger und ineinander übergehender Bewegungsformen.. Das aber ist speziell die theoretische Begründung für die Notwendigkeit gerade komplexer physisch-geographischer und wirtschaftsgeographischer.. Forschungen $\gg 13$.

18 Das direkte Gegenteil behauptet eine Gruppe von sowjetischen Wirtschaftsgeographen, von welchen W.S. WAssJUTIN und M. M. SchiRMUNSKi als Exponenten gelten können. Ersterer äußerte sich in einer Sitzung des Gelehrtenrates des Wirtschaftsinstituts an der Akademie der W'issenschaften der UdSSR (Mängel und Aufgaben der wirtschaftswissenschaftlichen Forschungsarbeit. Fragen der Wirtschaft 1948, Nr. 8, deutsch in "Sowjetwissenschaft “ 1949, Nr. 2, 3, 4), letzterer in diversen Aufsätzen, von denen hier zwei zitiert seien: Zum Gegenstand der ökonomischen Geographie als Wissenschaft (Nachr. d. Akad. d. Wiss. UdSSR, Geogr. Serie 1951, Heft 3, deutsch in „Sowjetwissenschaft 2, 1952, Gesellschaftswiss. Abt. und Zeitschr. für den Erdkundenunterricht 5, 1953, Heft 1/2) und" Die geographischen Wissenschaften in der UdSSR (Manuskript 1952, in dankenswerter Weise vermittelt durch die Gesandtschaft der UdSSR in Bern). Wir zitieren im folgenden nur SchiRmunski. Nach ihm resultiert die Wirtschaftsgeographie aus der Notwendigkeit, innerhalb der Wirtschaftswissenschaft Standortforschung zu betreiben. „Eine derartige Standortforschung wird Gegenstand einer besondern Wissenschaft, der Geographie der Wirtschaft bzw. der ökonomischen Geographie “. Damit ist bereits gesagt, daß die sowjetische Wirtschaftsgeographie gar nicht Geographie, sondern Wirtschaftsforschung, genauer Ge-Ökonomie ist, weil ihr Objekt nicht die Erde (Landschaft), sondern die Wirtschaft ist. Und SchIRMUNsKI stellt dies auch noch selbst ausdrücklich fest, wenn er sagt: „Die ökonomische Geographie untersucht also die konkreten Formen, den Charakter und die Gesetzmäßigkeiten der Verteilung der Produktion (d.h. eines Zrweiges der Wirtschaft, womit schon Einseitigkeit besteht, insofern ja auch der Konsum in diese hineingehört und geographischer Betrachtung bedürftig ist) in ihrer historischen Entwicklung und im Zusammenhang damit den Zustand und die Struktur der Wirtschaft eines bestimmten Landes oder Bezirkes... (sie) ist 1. eine Wissenschaft, welche die Bedingungen und Formen der pesellschaftlichen Entwicklung erforscht, d. h. eine Sozialwissenschaft, 2. eine der Sozialwissenschaften, die sich speziell mit den Entwicklungsbedingungen und 
Während nämlich noch im Sowjetzeitalter der führende und in den methodologischen Diskussionen immer wieder zitierte Geograph L. S. BERG die Auffassung

Formen der gesellschafttlichen Produktion befassen, d. h. eine ökonomische Wissenschaft “. (Weshalb sie dann trotzdem Geographie genannt wird, bleibt unerfindlich). Den uns hier besonders interessierenden Gegensatz zur physischen Geographie leitet SchIRMUNSKI aus dem dialektischan Materialismus ab. „Dieser stellt die allgemeinen Entwicklungsgesetze fest, die sowohl in der Natur als auch in der Gesellschaft herrschen. Die Ausdehnung eben dieser Thesen des dialektischen Materialismus auf die Erforschung des gesellschaftlichen Lebens... deckt die besondern Gesetzmäßigkeiten der gesellschaftlichen Entwicklung auf, die mit den die Entwicklung der Naturerscheinungen bestimmenden Gesetzmäßigkeiten nicht identisch sind. Dieser grundlegende prinzipielle qualitative Unterschied grenzt auch die beiden Hauptgruppen der Wissenschaft, die Gesellschaftswissenschaften und die Naturwissenschaften voneinander ab. Die Geographie kann sowohl zu den Gesellschaftswissenschaften als auch zu den Naturwissenschaften gehören. Zu den Gesellschaftswissenschaften gehören z. B. die ökonomische, politische Geographie, die Geographie der Bevölkerung usw., zu den Naturwissenschaften die physische Geographie, die Geomorphologie, die Hydrologie, die Pflanzengeographie, die Biogeographie u.a. Aber gerade weil die zu den Gesellschaftswissenschaften und die zu den Naturwissenschaften gehörenden Zweige der Geographie von prinzipiell verschiedenen Entwicklungsgesetzen ausgehen, haben wir nicht das Recht, von einer, einheitlichen geographischen Wissenschaft, von der Geographie schlechthin zu sprechen. Es wäre irrig, die Einheit der geographischen Wissenschaften darin erblicken zu wollen, daß das Forschungsgebiet einer jeden einzelnen und aller zusammen angeblich die , Erfüllıng des Raumes' sei. Nicht die ,Erfüllung des Raumes' an und für sich und als Selbstzweck ist das Forschungsobjekt einer jeden geographischen Wissenschaft, sondern der für jeden von ihnen charakteristische Kreis von Erscheinungen der Natur oder Gesellschaft, d. h. qualitativ verschiedener Erscheinungen! " Diese aus der erstgenannten Arbeit ScHIRMUNSKIS stammenden Sätze werden an Prägnanz übertroffen von entsprechenden aus der zweiten: „Der MarxismusLenismus lehrt, daß die Natur und die Gesellschaft von verschiedenen Gesetzen beherrscht sind. Im ersten Fall sind es Naturgesetze, im zweiten Sozialgesetze. Es kann deshalb keine, einheitliche Geographie' geben: die physische und ökonomische Geographie, so sehr sie Bindungen verknüpfen, sind zwei durchaus verschiedene Wissenschaften; die erste ist eine Naturwissenschaft, die zweite eine ökonomisch-soziale Wissenschaft". SCHIRMUNSKI (wie auch seine Gesinnungsgenossen) verkennt also - die Geographie augenscheinlich als Standorts- und Verbreitungslehre auffassend - offenbar völlig, daß es der Geographie gar nicht darum gehen kann, die Differenzen und Analogien von Natur- und Mensch (Kultur) - oder etwas anderes gewendet, die Kongruenzen und Inkongruenzen der Areale der Natur- und Kulturphänomene - (die zumeist bestehende Inkongruenz der ,naturund kulturräumlichen " Einheiten z. B. ist längst bekannt und nur noch im Detail abzuklären, also im Grunde "kein “ Problem, wohl aber das Zusammenwirken zu kombinierten Einheiten!) festzustellen, sondern daß sie allein die Zusammenhänge, Konnexe, Korrelationen aller terrestrischen Sphären und die daraus resultierenden (höhern oder komplexen) Gefüge (Landschaften) zu erkennen hat, daß also das Spezifikum der Geographie weder in der , Raumerfüllung' noch in der getrennten Betrachtung der Einzelsphären besteht. Er übersieht zugleich, daß Standortsforschung keineswegs gleich Geographie zu setzen ist, und daß die Notwendigkeit einer Standortsforschung gar nicht unbedingt eine Geographie erfordert, sondern daß Standortsprobleme grundsätzlich im Rahmen der Wirtschaftswissenschaft selbst zu behandeln sind. Wenn er deshalb abschließend glaubt, sagen zu können, daß es sich wohl erübrige, „die Fehlerhaftigkeit “ der ganzen Konzeption L. S. BERGs, des Verfechters einer Einheitsgeographie (und der Wirtschaftsgeographie als eines Teils der Kulturgeographie) zu beweisen, so kann hierzu nur gesagt werden, daß er damit sich selbst ad absurdum geführt hat. - In diesem Rahmen ist noch eine weitere merkwürdige Ansicht SchiRmusskis festzuhalten: die Subsummierung der Bevölkerungsgeographie unter die Wirtschaftsgeographie. „Das (die Bevölkerung als Hauptproduktivkraft der Gesellschaft betrachtende) umfassende Forschungsgebiet muß einem besondern Zweig der Wirtschaftsgeographie zugewiesen werden, nämlich der Geographie der Bevölkerung “. D. h. also nichts mehr und nichts weniger als daß der „Mensch“ der Wirtschaft unterzuordnen ist, eine Forderung, die allerdings in der bisherigen Gesellschaftsentwicklung nicht nur der UdSSR eine den Menschen beschämende Verwirklichung erfahren hat.

Nicht zuletzt ist zu betonen, daß auch die physische Geographie grundsätzlich "Sozial“wissenschaft ist, - da sie es nicht weniger als die Anthropogeographie mit (anorganischen und organischen) Sozietäten: Böden, Gewässern, Luft,massen“, Pflanzen- und Tier,gesellschaften zu tun hat - und daß auch die Anthropogeographie (die ökonomische Geographie der Sowjets, die übrigens bisher merkwürdigerweise keine besondere Sozialgeographie zu führen scheinen) nicht ausschließlich Sozialwissenschaft ist, insofern sie nämlich auch den menschlichen Individuen, Persönlichkeiten, Aufmerksamkeit zu zollen hat und insofern auch die menschlichen Sozietäten u. a. Naturgesetzen unterstehen. (W.) Neuerdings (Nachr. d. Geogr. Ges. UdSSR 85, 1953, H. 2) erfolgte unter dem Titel „Noch einmal über die methodologischen Grundlageu der Wirtschaftsgeographie und der Kampf gegen die Überbleibsel des Hettnerismus" von M. S. WoLOBUJEW-ARTEMow ein weiterer scharfer Angriff auf die „Einheitsgeographie“. Da er jedoch keine grundsätzlich neue Argumente enthält, wurde auf ihn nicht eingegangen. 
vertreten hatte, daß physio- und anthropogeographische Betrachtungsweise Glieder einer und derselben Wissenschaft seien, «begann man... diese beiden Betrachtungsweisen scharf voneinander abzugrenzen und verga $\beta$, daß die Geographie eine Einheit ist 》.

Es kam sogar dazu, daß die geographischen Fakultäten als ausschließlich der Physiogeographie gewidmete Institutionen aufgebaut, die Wirtschaftsgeographie dagegen den Gesellschafts- und Wirtschaftswissenschaften angegliedert wurde. Die Tatsache, daß jedoch stets gegenteilige Strömungen auftreten, läßt erkennen, daß offenbar auch dieser Differenzierungsvorgang der öffentlichen Stellung der Geographie nicht durchwegs zum Heile gedieh.

Auch der Votant N. L. BLAGowidow vertrat die Ansicht, daß die Trennung von physischer und ökonomischer Geographie dieser Disziplin nicht förderlich gewesen sei. Ihm lag jedoch neben der Forderung nach erneutem Zusammenschluß beider vor allem daran, vor scholastischer, «formal-kartographischer » Interpretation der geographischen Wirklichkeit zu warnen und zu zeigen, daß SmIRNows und anderer Versuche, Landschaften und Rayons (die wirtschafts-geographischen Gebietseinheiten) durch scharfe Grenzen zu fixieren, zum vornherein zur Unfruchtbarkeit verurteilt gewesen seien, da (nach ENGELs) «starre und feste Grenzen überhaupt nicht mit der Entwicklungstheorie vereinbar sind, (vielmehr) alle Unterschiede in der Natur durch Zwischenformen ineinander über(gehen) »14. Entlang den Wasserscheiden «starre Grenzen zu ziehen ist Metaphysik!» Andrerseits plädiert Blagowidow, abermals im Gegensatz zu SMIRNow, für eine maximale Unterteilung und Differenzierung landschaftlicher Einheiten, indem er betonte, die Praxis, namentlich die Landwirtschaft, verlange ein Hinuntergehen bis zur Mikrolandschaft, was letzten Endes entscheide. Zugleich setzte er sich vehement für eine Faktorenlehre in der Landschaftsforschung ein, deren "Vernichtung ... als eines Erbstückes des Machismus 》SMIRNow gefordert hatte. "Natürlich kann die Lehre von den Faktoren... schädlich sein, wenn sie nur ein äußerliches Verhältnis des «Schiebenden» zum «Geschobenen» ausdrückt, wenn man die Wirkung der Faktoren als isoliert und einseitig annimmt. Aber eine solche Auffassung der Faktoren gibt es bei uns nicht... Wir müssen von dieser Kategorie Gebrauch machen, wenn wir der Philosophie Lenins und Stalins folgen wollen. Das Wort «Faktor» selbst soll uns nicht irre machen. Wir benutzen doch auch den Begriff «Materie », obwohl ihn vor uns schon naive Materialisten und Idealisten auf ihre Weise benutzt haben. Dasselbe trifft auch auf den Begriff «Landschaft» zu». Ihn gemäß Hettner oder im Geiste unserer Dokutschajewschen Geographie zu verstehen, die die festen Positionen des dialektischen Materialismus inne hat, sind zwei verschiedene Dinge, die man nicht.. verwechseln darf. Vom praktischen Gesichtspunkt ist die Lehre von den Faktoren nötig... Man muß zu einem quantitativen Ausdruck nicht nur der Faktoren selbst zu kommen suchen, sondern auch der Ergebnisse ihres wechselseitigen Zusammenhanges. Man muß den Begriff der bestimmenden und fördernden (bezw. hemmenden) Faktoren entwickeln (wobei man die Veränderlichkeit der Wirkungskraft der Faktoren im Raum, in der Zeit, in verschiedenen Kombinationen zu beachten hat). Außerordentlich groß ist dabei die

14 Vgl. hierzu das Zitat des Chemikers W. Ostwald: „der Mangel ganz vollständiger und eindeutiger Grenzen (ist) eine ganz allgemeine Erscheinung bei allen natürlichen Dingen, wie die Wissenschaft auch eines ist " (Grundriß der Naturphilosophie, Leipzig 1908. p. 10). Diese Ansicht ist geeignet, einen Albdruck von jenen Geographen zu nehmen, die in der Unmöglichkeit deutlicher Begrenzung ihres Forschungsobjekts eine crux der Geographie erblicken. Im Grunde bedeutet indes die Schwierigkeit der Objektbegrenzung in der Geographie einen eigentlichen Vorzug dieser Disziplin. Symbolisiert doch der Mangel scharfer Grenzen der Landschaft (bei denen überdies sehr einseitig meist nur in der Horizontalen gedacht wird, während"die nicht weniger wichtigen Grenzen in der Vertikalen: gegen den Kosmos und das Erdinnere sowie die Sach- und Zeitgrenzen [Wann hört eine Landschaft z. B. auf, entwicklungsmäßig die gleiche zu sein, wann ist am selben Ort von einer "neuen “ zu sprechen?] meist außer Acht gelassen werden) geradezu einen besonders hohen Realitätsgrad des geographischen Objekts. (W.) 
Rolle des geographischen Verfahrens, welches die Wechselwirkung der Faktoren synthetisch darstellt ». Von der richtigen Beurteilung der Faktoren im Gesamtgefüge der Landschaft verspricht, sich BLagowidow durchgreifende Anwendungsmöglichkeiten auf deren Gestaltung, die ihm wie seinen Kollegen eigentlichstes Ziel der Geographie ist, wobei er, vielleicht noch schärfer als jene, die positive Bedeutung von vorsowjetischen Geographen (Dok utschajew, Berg u. a.) würdigte ${ }^{\mathbf{1 5}}$.

Die Voten seiner Vorredner mehr oder weniger bekräftigend, wiederholte auch der letzte Redner des Gesprächs, W. W. Newski, im wesentlichen die Punkte, die im vorangehenden zitiert worden sind. Es sollen daher hier nur noch einige Formulierungen angeschlossen werden, die diese besonders einprägsam und bezeichnend zu beleuchten scheinen. So erinnerte NewsKI daran, daß «nach ENGels die Selbständigkeit einer Wissenschaft dadurch bestimmt wird, daß jede.. eine Bewegungsform der Materie oder einige untereinander verbundene Formen untersucht. Auf der Erdoberfläche beobachten wir gesetzmäßige Gruppierungen der Gegenstände und Erscheinungen. Diese... sind nichts Sichselbstgenügendes. Sie hängen eng miteinander zusammen und bilden komplizierte Komplexe, die den Namen Landschaften erhalten haben (und die zu studieren die Geographie unternommen hat). Keine andere Wissenschaft beschäftigt sich mit diesen ganzheitlichen Naturkomplexen. Wir kennen Naturkomplexe verschiedenen Komplexheitgrades: Landschaft, geographische Zone, geographische Sphäre der Erde (Gesamtheit aller Landschaften, W.). Aber weil sie alle einen und denselben (Grund-)Charakter tragen, müssen sie auch Objekt einer einzigen Wissenschaft sein. Der Zweig der Geographie, der die geographische Sphäre im ganzen untersucht, heißt allgemeine Erdkunde $(\mathrm{m}$. E. richtiger Globalerdkunde, W.); die Erforschung der einzelnen Teile (Regionen) dieser Sphäre ist der Aufgabenbereich der regionalen Erdkunde (Landschaftskunde, Länderkunde). Mit dieser Ansicht stimmen die meisten sowjetischen Geographen überein. Aber in der Praxis liegen die Dinge nicht immer so wie in der Theorie. Wenn man sich beispielsweise den Lehrbüchern zuwendet, muß man feststellen, daß bis vor kurzem der Abschnitt «Allgemeine Erdkunde» nur dem Namen nach existierte, in Wirklichkeit aber keine Wissenschaft war, sondern die enzyklopädische ${ }^{16}$ Vereinigung einer Anzahl Wissenschaften.... Die Behauptung des Genossen SMIRNow, alle sowjetischen Geographen seien vom Geiste HeTtNers angesteckt, stimmt nicht... (sie) haben schon längst mit Hetrner gebrochen. Die Tatsache, daß3 wir das Wort «Landschaft» verwenden, das auch bei HeTTNeR in Gebrauch war, bedeutet gar nichts. HeTTNNER leugnete die Existenz von Landschaften in der Natur und betrachtete die Landschaft als eine für die Gliederung der Natur bequeme (willkürliche) Kategorie ${ }^{17}$. Die Sowjetgeographen haben dem Begriff Land-

15 So sehr dieser ja gleichfalls bei uns diskutierten Forderungen nach einer Faktorenlehre grundsätzlich beizupflichten ist, so deutlich muß doch auch hier betont werden, daß eine solche wirklich Faktorenlehre: Lehre von den Einzelerscheinungen der Erdoberfläche nicht im Sinne von (selbständigen) Einheiten, sondern allein und ausschließlich im Sinne von Teilen, Gliedern, Gestaltungselementen, eben Faktoren der Landschaft sein muß, wenn sie nicht aus der Geographie herausfallen soll. Denn so evident scheint, daß das Landschaftsganze (wie jedes Ganze) olıne seine Teile nicht denkbar ist, so unbestreitbar dürfte andrerseits sein, daß Erkenntnis dieser Teile im Sinne selbständiger Einheiten oder Phänomene für sich selbst dann keine Landschaftserkenntnis ergibt, wenn deren Verbreitung oder (räumliche) Anordnung auf der Erde, ihre regionale Eigenart oder die von ihnen gebildeten Areale (z. B. Relief-, Klima-, Wirtschafts-Gebiete usw.) untersucht werden. (W.)

16 Allerdings auch ein scheinbar so heterogenes Gebilde wie eine Universal-Enzyklopädie (Brockhaus, Meyer, Sowjetenzyklopädie usw.) läßt sich als ein „einheitliches Ganzes“, d. h. als eine zusammengesetzte in sich geschlossene zusammenhängende gegen außen als „Eins" als „Einheit" erscheinende Mannigfaltigkeit auffassen!

17 Offenbar kannte Newski weder A. Hetrners Aufsatz „Der Begriff der Ganzheit in der Geographie " (Geogr. Zeitschr. 40, 1935, Heft 4) noch dessen nicht weniger eindeutige ältere Abhandlungen „Geographische Anschauung “ (Daselbst 19, 1913, H. 1, wo er abschließend sagte..." der Nachweis, daß die Natur der Länder kein zufälliges Neben- und Durcheinander von Erscheinungen, 
schaft einen prinzipiell neuen Inhalt beigelegt und haben vor allem festgestellt, daß die Landschaften real in der Natur existierende Gebilde sind. Allein Genosse Smirnow hat sich von der Natur abgewandt, blickt Hettner in die Augen und spricht ihm nach, daß Landschaften nicht existieren und bei dieser Gelegenheit nennt er jeden sowjetischen Geographen einen Idealisten, der das Wort « Landschaft 》erwähnt. Von den Landschaften dürfen wir nicht abgehen, weil sie eine objektiv existierende Realität sind. Wer eine ... Realität erforscht, kann kein Idealist sein. Entgegen Genossen SMIRNow sprechen die sowjetischen Geographen nicht von der Einheitlichkeit..., sondern vom typischen Charakter der Landschaft. Das sind verschiedene Dinge, die man nicht durcheinander bringen dürfte ».

So ergab die Diskussion so gut wie einhellig, daß die Sowjetgeographen an der Landschaft als an einer grundlegenden Kategorie und einem realen Objekt ihrer Wissenschaft festhalten wollen und dies auch in gewissem Sinne in überzeugender Weise zu begründen verstehen. Hierbei ist Landschaft immer als ein «charakteristisches Teilstück der Erdoberfläche» verstanden, das sich qualitativ von andern Teilstücken unterscheidet, natürliche Grenzen besitzt und eine ganzheitliche, wechselseitig bedingte, d. h. gesetzmäßige Anhäufung (Kombination) von Gegenständen und Erscheinungen darstellt (vergl. S. 23i). "Jede Landschaft verhält sich zur geographischen Sphäre (Gesamtheit aller Landschaften der Erde) wie ein Teil zum Ganzen. Wie die geographische Landschaft von der geographischen Sphäre untrennbar ist, da sie einen Teil derselben bildet, ist auch die geographische Sphäre untrennbar von der Landschaft, da sie in und durch die Landschaften in Erscheinung tritt. Daher ist die Behauptung, die Geographie sei die Wissenschaft von der geographischen Sphäre, im Grunde gleichbedeutend mit der Behauptung, sie sei die Wissenschaft von den Landschaften. Aber die zweite Formulierung ist weniger gut, weil bei ihrer Verwendung die Gefahr entsteht, sich die Landschaft als autonomes System vorzustellen, während dies in Wirklichkeit nicht der Fall ist. Den Charakter der wechselseitigen Zusammenhänge und folglich auch der Wechselwirkungen zwischen den Gegenständen.. der geographischen Sphäre (der Gesamtheit aller Landschaften) bezeichnet man passend als Struktur der geographischen Sphäre (oder der Landschaften). Die Struktur macht die innere Eigentümlichkeit jeder Landschaft und der geographischen Sphäre im ganzen aus. Von der Struktur und dem stofflichen Gehalt der geographischen Sphäre hängen sowohl ihr äußeres Aussehen (Morpholo-

sondern ein gesetzmäßiges und harmonisches Ganze ist, muß ein reicher geistiger Gewinn ... sein “) und "Geographische Forschung und Bildung " (Daselbst I, 1895, Heft I, in der er programmatisch betonte: „Die... Erscheinungen einer Erdstelle sind ursächlich eng miteinander verbunden und machen jede Erdstelle dadurch zu einer natürlichen Einheit, der man Eigenart oder Individualität zusprechen kann ... gleichsam... ein harmonisches Kunstwerk..."). War darnach HETINER ein Materialist oder ein Idealist (Metaphysiker), ein Leugner oder Bejaher der Landschaft als eines realen einheitlichen Objekts? Die Antwort darauf dürfte wohl ein jeder sich selbst geben können. $\mathrm{Daß}$ im übrigen aber doch auch "westliche" Geographen glauben, die Landschaft sei nichts "Gegebenes", „Vollkonkretes", sondern (willkürliche) „Setzung“", belegen Aussprüche wie "Die Gegenstände der Geographie sind nicht gegeben, sie müssen durch Geistestätigkeit erst gebildet werden (O. Schlóter, Geogr. Abende, Berlin 1919, 5, p. 32) oder „An sich ist sie (die Landschaft) nicht vorhanden, es gibt nur die wechselvolle Vielfalt der Erdoberfläche ... . ich zweifle daran, ob schlechthin gegebene natürliche Landschaften je gefunden werden ..., die voll real und nicht nur... abstrakt-real sind“ (H. SchmitThenner, Geographica Helvetica VI, 1951, p. 126 f.). Demgegenüber wird hier, mit KaLESNIK, die These vertreten, daß Landschaften so "gegeben“, „vollreal“ sind wie ihre

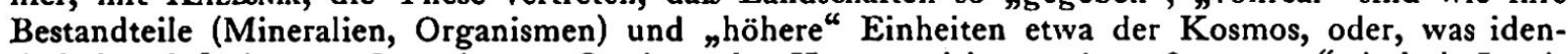
tisch ist, daß Atome, Organismen, Gestirne, der Kosmos nicht weniger „Setzungen“ sind als Landschaften; denn weder diese noch jene sind im Grunde mehr als Namen, Termini, Symbole für Sinneseindrücke, die jedoch „realen Dingen “ entsprechen oder entsprechen dürften, ohne daß wohl jemals zureichend ausgemacht werden kann, ob dem faktisch so ist. Anders ausgedrückt: Wir können von einer bestimmten Landschaft (ob sie nun scharf begrenzbar ist oder nicht) grundsätzlich "genau“ so wenig sicher oder so sicher sagen, sie sei real wie von einem bestimmten Bleistift, Hund oder Stern, es wäre denn, daß sich nachweisen ließe, daß zwar diese Dinge konkret bestünden, m. a. W. daß Landschaftsbildner Konkreta wären, Landschaften selbst dagegen nicht. (W.) 
gie, Form, Bild bei N. A. Solnzew) als auch die Eigenart ihrer innern Entwicklung ab. Der Entwicklungsprozeß seinerseits erzeugt auch eine neue Struktur sowie ein neues Aussehen, weil sowohl das eine wie das andere stets das Ergebnis der vorangegangenen Entwicklung ist.... Den gesetzmäßigen Strukturwechsel, die Entwicklung der geographischen Sphäre, kann man als geographischen Prozeß bezeichnen. Dieses Wort ist jetzt einer sehr scharfen Kritik unterzogen worden, weil Akademiker GrigorJEW unter Prozeß nicht Entwicklung versteht, sondern lediglich die Wechselwirkung der Gegenstände und Erscheinungen. Aber wenn man in den Terminus «Prozeß» den Begriff der Entwicklung des geographischen Milieus hineinlegt, dann begegnet man kaum noch begründetem Widerstand. Es ist klar, daß die Entwicklung der geographischen Sphäre nicht das mechanische und zufällige Ergebnis der Entwicklung ihrer einzelnen Teilstücke und Bestandteile ist. Obwohl sich jeder Bestandteil (Relief, Böden, Vegetation bsw.) nach seinen eigenen, nur für ihn charakteristischen Gesetzen verändert, verläuft doch infolge engster Berührung, wechselseitiger Bedingtheit und gegenseitiger Einwirkung dieser Teile, die zusammen die geographische Sphäre bilden, die Entwicklung derselben wie die Entwicklung eines ganzheitlichen Naturgebildes. Das bedeutet, da $\beta$ wir, wollen wir den Terminus «geographischen Prozeß» beibehalten, sagen müssen, da $\beta$ der geographische Prozeß durch Komplexheit, innere Widersprüchlichkeit und zugleich durch Einheitlichkeit charakterisiert wird. Und das ist... die richtige Behandlung des Begriffes des ... geographischen Prozesses. Hier liegt die Analogie zum Lebensprozeß... und zu dem einheitlichen Prozeß der Bodenbildung, der, nach WiLjams, als ein Häufung von Prozessen aufzufassen ist, durch die sich auf dem Muttergestein der Boden entwickelt... Schließlich (ist noch zu unterstreichen), daß es bei der Erforschung der geographischen Sphäre... unbedingt erforderlich ist, den Einfluß der menschlichen Gesellschaft auf die Natur zu berücksichtigen... Ohne dies... kann es keine ernsthafte wissenschaftliche geographische Forschung geben... Man braucht (endlich) wohl kaum zu betonen, daß das geographische Milieu unter einem Gesichtswinkel erforscht werden muß, der zur Aufdeckung der maximalen Möglichkeiten für die Ausnutzung und Umgestaltung (desselben) führt 》 (KALESNIK).

Überblicken wir nun abschließend, unter Berücksichtigung auch anderer jedoch nicht ausgewerteter neuerer methodologischer Arbeiten der Sowjetgeographen die Situation unserer Wissenschaft in der UdSSR, so haben wir festzuhalten, daß diese offenbar aus verschiedenen Gründen prekär ist. Die wohl maßgebende Basis dürfte zweifellos die von den Sowjetgeographen selbst wie auch andernorts betonte Tatsache des Zurückbleibens hinter den "Anforderungen der Volkswirtschaft“ sein, die umso verhängnisvoller ist, als im Marxismus in der "Brauchbarkeit" einer Wissenschaft der Prüfstein für ihre Existenzberechtigung erblickt wird. Ob dieses Zurïckbleiben in der Tat, wie verschiedene Fachgenossen annehmen zu dürfen glauben, auf die mothodologischen Divergenzen zurückzuführen ist, muß hier unerörtert bleiben. Es erscheint dies immerhin insofern mindestens fragwürdig, als in den grundlegenden Fragen bei allen Sowjetgeographen Einhelligkeit herrscht: ist doch für alle die marxistische-leninistische(-stalinistische) d.h. dialektisch-materialistische Ideologie gemeinsamer Ausgangspunkt, alle sind sich in diesem Zusammenhang darin einig, daß der alleinige Sinn der Geographie im möglichst optimalen Dienst an der sowjetischen, sozialistischen Gesellschaft, geographisch gewendet in der Schaffung möglichst idealer Möglichkeiten für eine optimale Nutzung der Landschaft bestehen könne, und schließlich betrachten alle die Wirklichkeit (wie ihre konkreten Teilbereiche) als ein „mannigfaltig-einheitliches“ Ganzes, als einen komplexen - bis zu einem gewissen Grade hierarchischen statisch-dynamischen Zusammenhang, der (zugleich) einem "Prozeß" (einer Entwicklungsfolge) entspricht. Wenn so in den grundlegenden Fragen Einstimmigkeit herrscht, bleibt umso verwunderlicher, daß in Sekundärproblemen die Ansichten anscheinend so sehr auseinandergehen wie die Zitate erkennen lassen.

Versuchen wir rückblickend die Differenzen zu sichten, so erkennen wir, daß sie doch wohl nur in der verschiedenen Interpretation weniger Begriffe, insbesondere des Begriffes Landschaft gesucht werden können. Im Grunde geht es sogar lediglich darum, ob die von allen (physischen) Sowjetgeographen unbeanstandet als Oberbegriff der Geographie betrachtete „geographische Hülle “ oder "Sphäre“, (die Erdoberfläche der westlichen Geographen) mit Landschaft synonym verwendet werden kann, m. a. W. ob Landschaft und geographische Hülle als wesensanaloge Phänomene zu betrachten sind. Ohne intervenieren zu wollen, darf hierzu bemerkt werden, daß kein Grund besteht, 
die Frage zu verneinen. Denn der Landschaftsbegrift ist keineswegs räumlich fixiert, da vielmehr durchaus verschiedene Landschaftskategorien, z. B. Größenordnungen (Orts-, Bezirks-, Kontinental-, Meeres-, Globallandschaft) ebenso wie Landschaftsindividuen als auch -typen unterschieden werden können ${ }^{18}$. M. a. W. die "geographische Sphäre" läßt sich durchaus zu Recht als Gesamtheit aller "Einzellandschaften " niedrigerer Größenordnung wie als ein besonderes Landschaftsindividuum (eine Großlandschaft: Globallandschaft) auffassen. So bleiben innerhalb des Oberbegriffs Landschaft nur noch die Antinomien Realität und Idealität (Irrealität), Zufälligkeit und Notwendigkeit, Einheitlichkeit und Uneinheitlichkeit (bzw. Dualität, Heterogenität) als strittige Punkte, wobei je nachdem die Verfechter der einen oder andern Kategorie als „Pseudogeographen “ oder als Vertreter der wahren dialektisch-materialistischen Geographie erscheinen. Doch besteht keinerlei Zweifel darüber, daß auch diese Divergenzen sich unschwer beseitigen ließen. Dies zu tun steht indes nicht einem Einzelnen oder einzelnen Gruppen zu, sondern müßte Gegenstand einer (internationalen) Konvention sein. Der Entscheid aber hängt allein von der konventionellen Interpretation der strittigen Kategorien Einheit-Uneinheitlichkeit, Realität-Irrealität usw. ab, wozu auf Logik und Erkenntnistheorie und (damit) bis zu einem hohen Grade auf die Praxis des täglichen Wortgebrauchs abzustellen wäre. Uns genügt, an wenigen Zitaten gezeigt zu haben, daß offenbar in der Sowjetgeographie die gleichen methodologisch-ontologischen Fragen kontrovers sind und diskutiert werden wie anderswo und daß sie trotz einer der „, bourgeoisen Geographie“ bewußt entgegengesetzten dialektisch-materialistischen Auffassung der Sowjets auch grundsätzlich gleich zu lösen versucht werden (weil sie nicht anders zu lösen sind!) - schließlich nicht zuletzt, daß eine zureichende Lösung auf dem Wege der Konvention durchaus gefunden werden kann. Dabei ist anzuerkennen, daß den russischen Geographen vielfach Formulierungen gelungen sind, die uns bisher fehlten, während sie andrerseits freilich oftmals Auffassungen äußern, die in der übrigen Welt längst konventionelle Gültigkeit haben - was namentlich ältere Sowjetgeographen ja auch anerkannten (z. B. BERG). So bedauerlich, zusammengefaßt, die Kluft ist, welche auch auf geographischem Forschungsbereich die Sowjets von der Umwelt trennt und so unüberbrückbar sie angesichts der Haltung der Sowjetgeographen scheint (wobei die These des dialektischen Materialismus nicht weniger metaphysisch ist als jene des "Idealismus", nicht weniger bloß Behauptung statt Beweis darstellt und daher keineswegs vorurteilsloser Wissenschaft entspricht), so sehr ist zu hoffen, daß die Zeit nicht ferne sei, da eine sowohl im Interesse der geographischen Theorie als der Praxis liegende internationale Verständigung erzielt wird.

18 Die im Gegensatz dazu von H. Boвek, J. Sснmiтhüsen und H. Lautensach propagierte, wohl auf N. KREBS zurückgehende, ausschließliche Verwendung der Landschaft als Typenbegriff entbehrt zwingender Logik und realer Auffassung. Es gibt sowohl Landschafts- und Länderindividuen als -typen (und -normen) und diese Tatsache negieren, heißt ihr ins „Gesicht schlagen“. (Vgl. die Ansicht KaLESNIKS S. 3).

\section{LA DISCUSSION AU SUJET DU PAYSAGE CHEZ LES GÉOGRAPHES SOVIÉTIQUES}

Ces dernières années, des discussions sur la méthode géographique semblent être devenues plus fréquentes et plus vives en URSS. La raison principale pourrait bien être comme le font entendre même les géographes soviétiques, que la géographie est restée en arrière des exigences de la vie pratique, ce qui est attribué à l'insuffisance de la théorie de cette discipline. L'article ci-dessus cherche à éclairer cette situation, en s'appuyant sur une conférence de 1951 au sujet de la théorie de la géographie et sur d'autres discussions. On montre que la géographie est envisagée tantôt comme discipline unitaire, tantôt comme un groupement de disciplines qui font partie d'un côté des sciences naturelles, de l'autre des sciences sociales, celles-ci étant des sciences de rapports, celles-là des sciences de faits. En géographie physique, il existe aussi deux directions principales, dont l'une voit dans le paysage l'objet central, tandis que l'autre le considère comme un pseudo-concept, et ces adeptes comme des idéalistes et des métaphysiciens. Au-dessus de ces différences de principes, on ne doit pas l'oublier, les géographes. soviétiques sont tout à fait unis par la lutte en faveur d'un matérialisme dialectique et rejettent la géographie "occidentale» comme tombée sous la coupe du capitalisme.

\section{UNA DISCUSSIONE SUL VALORE DEL PAESAGGIO NELLA GEOGRAFIA NEI SOVIET}

Negli ultimi anni sembra che le discussioni di carattere metodologico-geografico siano diventate più frequenti e più dibattute negli URSS. Secondo le affermazioni dei geografi russi, una delle cause principali dovrebbe essere ricercata nel fatto che la geografia non è più in grado di sodisfare alle esigenze poste dalla vita pratica, situazione cristallizzatasi soprattutto in conseguenza alla insufficienza di chiarezza nei fondamenti teorici della disciplina stessa. L'articolo cerca di risolvere il quesito basandosi su una conferenza trattante problemi teoretici avvenuta nel 1951 e su altre discussioni. Si dimostra che la geografia vien considerata da un lato come una disciplina unitaria, da un altro come un complesso di discipline; talvolta legata alle scienze naturali, tal'altra alle scienze sociali, dove le prime vengono ritenute discipline di ricerca di relazioni mentre le seconde discipline oggettive. Anche nell'ambito della geografia fisica le opinioni sono contrastanti: gli uni considerano il paesaggio come oggetto principale, gli altri lo giudicano uno pseudoconcetto e ritengono i suoi cultori idealisti e metafisici. Nonostante queste divergenze sui principi, i geografi russi sono tutti concordi, coerenti difensori del materialismo dialettico, nel negare la geografia occidentale perchè subordinata al capitalismo. 